\title{
APORTACIONES METODOLÓGICAS A LA INVESTIGACIÓN BIOGRÁFICA, RELACIONADAS CON EL PROCESAMIENTO Y LA ORGANIZACIÓN DE LOS DATOS
}

\author{
Methodological contributions to biographical research, related to processing \\ and organising data
}

David Herrera Pastor

Fecha de recepción: 04/09/2016

Fecha de aceptación: 08/11/2016

RESUMEN: Este artículo pretende dar a conocer algunas aportaciones metodológicas que se hacen a la investigación biográfica. Dichas aportaciones derivan de la Historia de Vida llevada a cabo en torno al caso de Ahmed, un menor infractor que se convirtió en educador de menores tras experimentar un proceso de reforma juvenil. El artículo gira en torno a: 1) Las herramientas creadas en esta investigación para categorizar los datos de acuerdo a las principales tareas que la metodología biográfica requiere (construir la biografía y analizar la información). 2) La nueva manera que se ha desarrollado para materializar el modelo de 'Relatos Cruzados', que otorga un carácter más versátil a su aplicación. 3) El proceso de síntesis seguido para definir el listado de categorías de análisis. Además de explicitar dicho proceso, se comparten diversas fundamentaciones respecto de la naturaleza, finalidad y última expresión de las mencionadas categorías. 4) Al carácter inductivo de la investigación y a los distintos procesos a los que sometieron los datos, que permitieron que el foco de investigación fuese emergiendo progresivamente $\mathrm{y}$, como consecuencia, se definiese de manera muy precisa.

PALABRAS CLAVE: Metodología de investigación, Biografías, Categorización, Métodos Inductivos, Foco de investigación.

ABSTRACT: This paper aims to share several methodological contributions to the biographical research. They derive from a Life History project developed about the life of Ahmed, a young offender who became "educator of minors" after experiencing a rehabilitation process. The research was complex in that: a) New tools to categorize data have been created, according to the task demanded: to analyse the information and build the biography. b) The crossed-narrative model could not be implemented in an orthodox manner, thus an alternative was developed to extend the model's versatility. c) The synthesis process followed to set the list of analytical categories is shared, incorporating some foundations their nature, aims and end expression. d) The focus of the research was built very precisely as a result of the inductive procedure followed during the research.

KEYWORDS: Research Methodology, Biographies, Categorization, Inductive methods, Research focus. 


\section{Introducción}

Este es un artículo sobre metodología de investigación biográfica. A través de él se quieren compartir una serie de aportaciones metodológicas que se hacen a dicha investigación. Las aportaciones se derivan de la Historia de Vida desarrollada en torno al caso de Ahmed, un menor infractor que se convirtió en educador de menores tras experimentar un proceso de reforma juvenil.

Para construir la biografía se recurrió a diversas fuentes de información y se utilizaron distintas técnicas de recogida de datos. Entre las más destacadas: 1) se realizaron entrevistas a Ahmed (en numerosas ocasiones) y a diecisiete personajes que jugaron un papel significativo en su historia (jueza del caso, subdirector del centro de internamiento, trabajadora social del centro de internamiento, tutora de libertad vigilada, madre de acogida, etc.). 2) Fuentes documentales: se consiguieron los expedientes de Ahmed en el Juzgado de Menores y en la Dirección General de Justicia Juvenil. 3) Se consiguieron más de doscientas fotografías, la mayoría del periodo en el que nuestro protagonista estuvo en el centro de internamiento.

La manera en que los datos fueron procesados y organizados a lo largo de la investigación, permitieron/demandaron el desarrollo de distintas aportaciones al método biográfico. A continuación, se relacionan dichas aportaciones:

- La investigación biográfica requiere una categorización dual de la información. En este trabajo se han creado unas herramientas que permiten realizar esa doble tarea, se han venido a denominar Unidades Biográficas (UB) y Unidades de Análisis (UA).

- Se ha desarrollado una variante del Modelo de Relatos Cruzados. Dicha variante consiste en realizar el cruce de los distintos testimonios de manera no consecutiva, lo que amplía la versatilidad del modelo.

- Se hace un recorrido por el proceso de síntesis llevado a cabo en esta investigación a la hora de definir el listado de categorías de análisis. Y se comparte una reflexión acerca de su naturaleza, finalidad y expresión última.

- El diseño inductivo permite concretar un foco de investigación muy preciso. El foco de esta investigación no estaba definido desde el principio. Por el contrario, la investigación surgió a partir del interés del investigador por la temática y de todas las preguntas que surgieron tras conocer el caso de Ahmed. Sin embargo, dicho foco se fue forjando y definiendo en el transcurso de la investigación, conforme se iban atravesando los distintos estadios y se iba cuestionando la información que se iba conociendo.

Distintas conclusiones se presentan a partir de todo lo mencionado con el propósito de contribuir a la expansión de la metodología de investigación biográfica.

\section{Posicionamiento interpretativo de la investigación}

Supongamos que consideramos el caso de John, un chico de doce años, y supongamos que los antecedentes familiares son de pobreza; su padre era delincuente, su madre le rechazó, su barrio es marginal. Supongamos que el $70 \%$ de los muchachos con los mismos antecedentes se hacen delincuentes. ¿Significa esto que hay un $70 \%$ de probabilidades de que John sea un delincuente? De modo alguno. John es un ser único; con una herencia genética propia; su experiencia vital es tan solo suya. Su mundo contiene influencias únicas, desconocidas para el estadístico: tal vez una relación afectuosa con cierto profesor o unas palabras juiciosas en alguna ocasión por un vecino. Estos factores pueden ser decisivos y 
pueden truncar esas probabilidades porcentuales. No existe ese $70 \%$ de probabilidades en relación a John. Éste podrá ser o no un delincuente (Allport en Pujadas, 2002: 41).

Necesitamos comprender los procesos subjetivos a través de los cuales las personas dan sentido y construyen sus vidas (Monteagudo, 2010), ya que el ser humano no se puede reducir a un conjunto cerrado de reglas nomotéticas ${ }^{1}$. Ni se pueden realizar postulados generales sobre el ser humano y la sociedad de manera 'objetiva' y absoluta. "Por mucho que afinemos en nuestras escalas de análisis de los factores que condicionan el comportamiento humano, existe un factor subjetivo irreductible, de carácter procesual, azaroso, imprevisible, con el que hay que contar" (Pujadas, Op. cit.: 41-42). No quiere decir que no existan reglas sociales y códigos culturales específicos que medien en el desarrollo humano. Quiere decir que la realidad social se muestra reacia ante el ordenamiento rígido y la imposición de reglas unívocas de funcionamiento (Bofill, Penacho y Quinzá, 2006).

En el ámbito social es necesario romper con esa idea de que el/la sujeto es un mero objeto de las normas y de la sociedad para recuperar una visión humanista que atienda y considere a la persona de acuerdo a su naturaleza y en toda su complejidad. El ser humano es un ser cultural, que se configura como tal en virtud de las significaciones que desarrolla y en las que se desenvuelve. Para comprenderlo adecuadamente se necesita investigarlo atendiendo a las distintas lógicas que configura en la realidad en la que se encuentra.

Teniendo en cuenta que el trabajo de investigación que aquí se contempla se enmarca dentro de las ciencias sociales y más concretamente dentro del ámbito de la educación, combinando aspectos pedagógicos con disyuntivas y problemáticas sociales, parece coherente adoptar un posicionamiento interpretativo para llevar a cabo el estudio. De hecho, el método de investigación elegido es el método biográfico, cuyos principios y fundamentos tienen como punto de partida el enfoque hermenéutico.

\section{Fuentes de información y técnicas de recogida de datos}

El método de investigación biográfico se caracteriza por ser flexible y permitir el uso de distintos instrumentos de recogida de datos y recurrir a diversas fuentes de información. En esta investigación, las entrevistas y las fuentes documentales fueron las fuentes de información principales, junto con una cantidad importante de fotografías significativas. Además, se utilizaron otras fuentes y se recurrió a otras estrategias de recogida de datos. En la siguiente tabla se presenta un resumen de todas ellas:

Tabla 1. Resumen de las fuentes de información y de las técnicas de recogida de datos

- Entrevista biográfica:

. 5 al protagonista de la historia.

- A los siguientes personajes, que jugaron uno o varios roles significativos en el desarrollo de la historia:

- Hermano de Ahmed.

- Jueza que llevó el caso.

- Subdirector del centro de internamiento.

\footnotetext{
${ }^{1}$ Las ciencias nomotéticas son aquellas que tienen por objeto las ciencias de la naturaleza, que buscan estudiar procesos causales e invariables. Por el contrario, las ciencias cuyo objeto es el estudio de los sucesos cambiantes, como la Economía, la Sociología, el Derecho o la Historia, son llamadas ciencias idiográficas.
} 
- Trabajadora social del centro de internamiento, que más tarde se convirtió en jefa de Ahmed en su actual trabajo.

- Tutor de Ahmed en el centro de internamiento.

- Una educadora del centro de internamiento que más tarde se convirtió en miembro del Equipo Técnicoº ${ }^{2}$.

- Dos monitores del centro de internamiento que ahora son amigos de Ahmed.

- Una trabajadora del servicio doméstico del centro de internamiento que posteriormente se convirtió en 'Madre de Acogida' de Ahmed.

- La tutora de Ahmed durante la medida de libertad vigilada.

- Un técnico de libertad vigilada.

- Un compañero de trabajo, de piso y amigo de Ahmed.

- DAFO.

- Registro iconográfico (más de 200 fotografías) + entrevista al respecto.

- Información inesperada: Manuscrito elaborado por Ahmed ("Significado de mi trabajo") + Entrevista.

- Documentos oficiales:

- Expediente en el Juzgado de Menores.

- Expediente en la Dirección General de Reforma Juvenil (Junta de Andalucía).

- Legislación vigente durante el caso: Ley Orgánica 5/2000, de 12 de enero de 2000, reguladora de la responsabilidad penal de los menores.

- Cuaderno del Investigador

- Frutos de la persistencia: Informe del tutor del centro de internamiento (14 Mayo 2001).

- Devolución de la información preliminar (dos veces a Ahmed y una vez al resto de informantes).

- Biograma y Sociograma + Entrevista

Elaboración propia

\section{Procesamiento y organización de la información}

Tras explorar la mayoría de fuentes de información planteadas y habiendo obtenido una cantidad de datos muy abundante, se comenzó a organizar la información y procesar los datos.

\subsection{Categorización de la Información. Inicio}

Una vez transcrita la información se inició el proceso de categorización. El profesor Pujadas (Op. cit.) distingue en dicho proceso entre 'unidades de contexto' y 'unidades de registro'. Aunque él realiza dicha distinción en un estadio más avanzado de la investigación y asigna a cada una de esas unidades una naturaleza diferente de la que a continuación se plantea, fue revelador aquella distinción, pues realizar una categorización dual sobre los distintos fragmentos de información podía resultar propicio para la fase de investigación que se comenzaba. Teniendo en cuenta que el método biográfico exige dos tareas: 1) Construir la biografía y 2) Analizar los datos, se pensó que podía resultar adecuado hacer dicha categorización dual en virtud de ellas. De esa manera, la información se podría organizar

\footnotetext{
2 El Equipo Técnico es un órgano independiente del Sistema de Justicia Juvenil que tiene carácter interdisciplinar (se compone de un trabajador social, un psicólogo y un educador social) y cuya misión es estudiar individualizadamente cada caso para, entre otras cosas, asesorar al Juez y al Ministerio Fiscal cuando sea necesario.
} 
desde el principio en función de la finalidad a que se destinase.

En ese sentido, la categorización de cada documento empírico recabado se inició desgranando la información separadamente en virtud de lo que se vino a denominar: las 'Unidades Biográficas' y las 'Unidades de Análisis':

- Unidad Biográfica (UB). Comprende cada fragmento de información de interés biográfico. La unidad biográfica selecciona el trozo de testimonio que puede servir para construir la biografía. A través de ellas se identifican los temas comprendidos en cada registro, explicitando el vínculo que tiene con el relato biográfico en proceso de elaboración.

La biografía se arma conjugando las unidades biográficas destiladas de todos los documentos empíricos. Dicha biografía ha de ser un mosaico que represente de manera lógica y con sentido, la interpretación significativa que el investigador hace (a partir de la identificación, procesamiento y organización) de todas esas unidades.

- Unidad de Análisis (UA). Se considera unidad de análisis la expresión mínima de información susceptible de ser escudriñada en virtud de los propósitos de la investigación. Cada UA comprende contenido sustantivo de interés vinculado al objeto de estudio. Es el segmento de información más elemental que se establece a la hora de armar el listado de 'categorías de análisis'.

La categorización consiste en "de-construir" cada registro de información en virtud de la naturaleza de las unidades que se estuviesen localizando. De este modo, todos los datos recabados se procesan para tratar de extraer de ellos las unidades biográficas y las unidades de análisis que corresponda. Siguiendo este procedimiento, en esta investigación todos los documentos empíricos fueron revisados dos veces ${ }^{3}$, una por cada tipo de 'unidad' planteada. Para ello, cada fragmento de información, que, a juicio del investigador, comprendía una unidad, se identificaba insertando un comentario que lo acotaba y describía en el lateral del texto. Dicho comentario se numeraba automáticamente siguiendo una lógica natural ascendente $(1,2,3$, etc.) y contemplaba la idea esencial que se desprendía del fragmento de información en cuestión. Las unidades de análisis solían ser desgranadas de manera literal, puesto que, en general, habían de ser las evidencias sobre las que, posteriormente, se había de articular la interpretación de los datos ${ }^{4}$.

Una vez finalizado el escrutinio del documento (entrevista, capítulo del expediente, etc.), se hacía un listado de todas las unidades extraídas, indicando la página en la que se ubicaba cada una de ellas. De ese modo las unidades resultaban fácilmente localizables. A continuación, vemos un fragmento de los listados de unidades biográficas y de análisis desprendidos de una entrevista tomada como ejemplo.

\footnotetext{
3 Revisar todo el material empírico en dos ocasiones permite, si se consideraba oportuno, añadir, eliminar o modificar alguna unidad, así como poner en tela de juicio y/o corroborar la labor realizada. En definitiva, aporta mayores garantías al proceso de categorización.

${ }^{4}$ En general, las unidades biográficas solían ser más numerosas y extensas y las unidades de análisis más concisas y directas.
} 
Tabla 2: Unidades Biográficas de la entrevista Ahmed 5

\section{UNIDADES BIOGRÁFICAS}

1. Estuvo en la escuela desde los 7 hasta los 12 ó 13 años. En ese sentido tenía dos madres [su madre y su hermana]. Su hermana, la mayor de todos los hermanos, prestaba mucha atención a la educación de los hermanos pequeños (Entrevista Ahmed 5, pp. 1-2).

2. La madre de Ahmed siempre se ha dedicado a las tareas del hogar y al cuidado de los hijos. El padre trabajaba en una fábrica de gravilla y también estuvo mucho tiempo en Gibraltar (Entrevista Ahmed 5, p. 2).

3. Aprovechó para dejar el colegio cuando su hermana se casó (Entrevista Ahmed 5, pp. 2-3).

Elaboración propia

Tabla 3: Unidades de Análisis de la entrevista Ahmed 5

\section{UNIDADES DE ANÁLISIS}

1. Empecé a trabajar, ya mi hermana se había casado, aproveché la oportunidad y dejé el colegio, porque antes, si no se hubiera casado yo no me hubiera podido dejar el colegio porque no hubiera querido (Entrevista Ahmed 5, p. 2).

2. Mi padre siempre es más duro y mi madre pues más, como él es más duro pues ella intenta siempre ser la más flexible (Entrevista Ahmed 5, p. 3).

Elaboración propia

Se trataba de un proceso de reducción de información que pretendía seleccionar, del sustrato empírico recabado, todos los datos relacionados con el objeto de estudio y hacerlos manejables en virtud de las tareas que había que realizar.

Cada unidad de análisis se refleja o está contemplada en una unidad biográfica, sin embargo, no ocurre lo mismo, al contrario. No todas las unidades biográficas tienen una unidad de análisis homóloga dónde se contemple la esencia de su contenido.

\subsection{La construcción de la biografía}

Construir la biografía eje de la historia de vida es una empresa compleja. Cortés y Medrano (2007) consideran que la representación de los datos resulta un aspecto complicado. No se puede caer en una inmediatez descriptiva que se deje llevar por el orden sugestivo de los testimonios. La biografía es una construcción que tiene su propia naturaleza y requiere de una lógica, una coherencia, una contextualización (Kushner, 2002; Roberts, 2002; Waller y Simmons, 2009), etc. que la hagan inteligible y proporcionen sentido a la narración (Arnaus, 1995). Como dicen Marinas y Santamaría (en Bolívar, Domingo y Fernández, 2001: 37-38) es necesario hacer que el relato "le devuelva la vida a la historia misma".

La construcción de la biografía quería desarrollarse siguiendo el modelo de relatos 
cruzados $^{5}$, es decir, se pretendía que la matriz de la biografía fuese el relato del protagonista de la historia, dividido en diferentes capítulos (que su voz diese cuerpo a la estructura de la narración). Y que las expresiones en tercera persona se insertasen tras el testimonio de Ahmed, comprendiendo la parte final de cada capítulo. Dichos testimonios vendrían a complementar y/o contradecir las expresiones del actor principal, de manera interrelacionada. En definitiva, se quería configurar la narración a partir del diálogo establecido entre las distintas voces, que interaccionando con fluidez otorgarían a la lectura vida y dinamismo.

"El personaje central de la narración debido a su singularidad y relevancia, nos ofrece la melodía, mientras los otros relatos nos ofrecen la armonía, el contrapunto, la fuga. Una estructura compleja, que enriquece el relato original, que lo enmarca, lo sitúa en perspectiva y que, a efectos analíticos, separa los factores más idiosincrásicos de la visión de la situación, para restituirnos un discurso multicentrado que gana en profundidad y en objetividad" (Pujadas, Op. cit.: 56).

Como la voz del protagonista había de constituir la matriz de la biografía, la construcción del relato biográfico se inició armando en primer lugar lo que se denominó el 'Relato en Primera Persona' (R1 o R1a). Dicho relato estuvo compuesto por informaciones desprendidas directa y únicamente por Ahmed. Su construcción se inició compilando en un mismo documento todas las unidades biográficas desprendidas de las entrevistas y de las fotografías facilitadas por él.

Aquel relato había de ser el eje sobre el que cruzar el resto de voces e informaciones significativas (sistema polifónico) que ayudaría a comprender la historia de manera holística y exhaustiva.

\subsubsection{Dilema metodológico}

Una vez se había realizado aquella labor, se emprendió la tarea de cruzar los relatos. En el desarrollo de dicha tarea se planteó el siguiente dilema metodológico:

- En aquel momento de la investigación no se sabía de qué información se podía prescindir. No se había definido el árbol de categorías de análisis, por lo que se podía prescindir de ningún dato. Lo que obligaba a mantener toda la información habida.

- Por otro lado, la información en tercera persona era ingente. Intentar incorporar aquel compendio de datos tan numeroso desvirtuaba la esencia del método de relatos cruzados. Insertar en cada capítulo, tras el testimonio de Ahmed, una cantidad de información tan enorme, alteraba la esencia del modelo planteado: lo hacía menos interactivo y dinámico, y robaba el protagonismo al actor principal de la historia.

Aunque se consideraba que lo más adecuado era armar la biografía de acuerdo al modelo de relatos cruzados, parecía que las particulares circunstancias del caso no se ajustaban de manera ortodoxa a aquel modelo. ¿Qué se podía hacer para superar aquella situación? Para tratar de salir de aquella disyuntiva se plantearon las dos opciones siguientes:

- Por un lado, hacer el cruce de los testimonios a posteriori, es decir, confrontar las distintas informaciones una vez se hubiesen definido las categorías que regirían el análisis de la biografía. De ese modo, se sabría con precisión qué fragmentos

\footnotetext{
5 Oscar Lewis es el autor de "Los hijos de Sánchez" que es la obra que dio a conocer el modelo de Relatos Cruzados internacionalmente. En España, el profesor Juan Gamella tiene una obra titulada: "La historia de Julián. Memorias de heroína y delincuencia (1990) que utiliza dicho modelo y resulta una auténtica obra maestra.
} 
resultaban significativos (y cuáles no) de cara a la interpretación de los datos. Y se podría armar un relato biográfico coherente con el foco de la investigación.

De lo contrario, si se seleccionaban los segmentos de evidencias que habían de completar la historia previa elaboración del listado de categorías, se podía incurrir en algunos problemas: a) El investigador podía actuar de manera arbitraria a la hora de seleccionar los fragmentos y armar la historia. b) Se podría descartar información relevante. c) No se garantizaría la rigurosidad del procedimiento, etc.

- Por otro lado, se tenía la opción de armar un 'Relato en Tercera persona' que diese cabida a toda la información que no provenía directamente de Ahmed, sin tener que descartar ningún dato en aquel momento. Ese texto se presentaría tras el 'Relato en $1^{\mathrm{a}}$ persona', siguiendo una estructura de capítulos similar a la desarrollada en la primera parte de la historia, de manera, que se pudieran establecer paralelismos y contraste entre las informaciones provenidas de cada fuente.

Sin tomar una decisión por ninguna de las dos opciones, se decidió salir de aquel pequeño bache, revisando una por una todas las transcripciones de los personajes secundarios y utilizando las unidades de análisis de dichas entrevistas como guía para convertirlas en un texto narrativo contextualizado donde se sintetizase la información más sustantiva de cada una de ellas. Ese nuevo documento se re-ordenó de acuerdo a la estructura de capítulos establecida en el Relato en Primera Persona. Por ejemplo, si la transcripción de la entrevista a la trabajadora social del centro de internamiento tenía originalmente 14 páginas, tras la síntesis se quedó en 5 de información sustancial que se vinculaba a los siguientes capítulos de la narración:

- Cap. V. En el centro de internamiento

- Cap. VII. El paso a la libertad vigilada y la finalización del proceso de reforma

- Cap. VIII. Un puente hacia la vida real

- Cap. IX. Educador de menores en protección

- Epílogo

Tras darle aquella segunda vuelta a la información, cada registro (de los personajes secundarios) tuvo localizada la información seleccionada y organizada, correspondientemente, en virtud de los capítulos establecidos. De aquella manera la información resultaba mucho más versátil y manejable de cara a la construcción de la biografía.

Paralelamente, se hizo un resumen narrativo de la información que se iba a utilizar de cada personaje secundario. Dichos resúmenes permitían hacer una mejor composición de lugar de cada testimonio, de acuerdo a la fuente de información y en relación con la biografía.

Sendas tareas (los "Fragmentos para el relato" y los "resúmenes a los personajes secundarios") ayudaron a visibilizar toda la información de una manera más gráfica y clara para poder terminar de construir el relato biográfico, es decir, insertar las voces de los personajes secundarios y demás informaciones en la biografía adecuadamente. Realizar aquellas dos tareas también sirvió para revisar que ninguna información relevante quedase desatendida. 


\subsubsection{Justificación de la utilización del método de relatos cruzados de manera no consecutiva}

Una vez se finalizó aquella segunda revisión de los registros, parecía haber unas mejores condiciones para terminar de redactar la biografía.

Finalmente, se decidió solucionar el dilema tomando la segunda opción planteada, es decir, armando un 'Relato en Tercera Persona' (R3 o R3a) que diese cabida a toda aquella información que podía ayudar a comprender de una manera más exhaustiva la biografía (testimonios de los personajes secundarios, sus DAFO, documentos de los expedientes de Ahmed: en el Juzgado de menores y en la Dirección General de Reforma Juvenil, "Ley del Menor" e informe del tutor de Ahmed durante la medida de internamiento). Dicho R3a debía aparecer en la biografía tras el 'Relato en Primera persona' siguiendo su misma estructura de capítulos ${ }^{6}$. La biografía, por tanto, quedó conformada a partir de dos relatos: el 'Relato en $1^{\text {a }}$ persona' y el 'Relato en $3^{\text {a }}$ Persona'.

Aunque en el modelo de relatos cruzados el cruce de los testimonios se realizaba de manera consecutiva en cada capítulo, en esta investigación el cruce de la información se ha tenido que hacer de manera no consecutiva.

El inconveniente era que la narración no iba a ser tan dinámica e interactiva como se pretendía. Y las ventajas, que se podían contrastar y establecer inferencias entre los datos de capítulos homólogos y que no había que desprenderse de ningún tipo de información. Lo que posibilitaba cruzar una cantidad de información más amplia que permitía una visión holística (que abordaba prácticamente todas las dimensiones del vivir de su protagonista) y exhaustiva (detallada y profunda) del caso. Además, aquel formato ubicaba, claramente, el testimonio de Ahmed en un lugar protagonista, el mismo que como "menor infractor" jugaba dentro del proceso de reforma juvenil. Lo que contribuía a desarrollar uno de los propósitos planteados en este trabajo con el método biográfico: otorgar voz a quienes, por diversas circunstancias, se suelen encontrar en situación de invisibilidad.

Construir la historia de aquella manera iba a permitir conocer de manera más precisa al protagonista de la investigación, ya que, como se ha dicho, la información que se utilizó para construir el primer relato se había desprendido únicamente de Ahmed, lo que permitía visionar la interpretación que el protagonista hacía de su propia historia, sin que dicha interpretación estuviese mediada por los testimonios de terceras personas. Eso fue un aspecto importante en el desarrollo de la investigación porque las características personales de nuestro protagonista fueron una clave del análisis del caso.

\subsection{Categorización de la Información. Continuación}

Una vez se había redactado la biografía había que centrarse en el análisis de la información. En aquella fase, el propósito era definir un listado de categorías de análisis que permitiese escudriñar toda la información sustantiva comprendida en el relato biográfico, "sin descontextualizarla y procurando ser fiel a los datos" (González y Padilla-Carmona, 2014: 89). Concretamente, se trataba de crear una estructura donde tuviesen cabida las evidencias susceptibles de ser examinadas en virtud de los intereses de la investigación. La sistematización de la información había de guiar al investigador en el análisis del foco de estudio.

\footnotetext{
${ }^{6}$ Si bien el 'Relato en $3^{\text {a }}$ Persona' no cuenta con el 'Capítulo X. Más allá de una relación de amistad'. Dicho capítulo aparece únicamente en el 'Relato en $1^{\mathrm{a}}$ persona'. En él Ahmed cuenta cómo se desarrolló su relación sentimental con una chica que conoció en aquella época. No se armó el homólogo en el 'Relato en $3^{\text {a }}$ Persona', porque no se consideró relevante de cara al estudio.
} 
En esta investigación el proceso se llevó a cabo de manera inductiva y emergente. Las categorías de análisis se conformaron a partir del proceso de síntesis de la información (Rivas, Herrera, Jiménez y Leite, 2009).

Los datos cualitativos que extraemos de una historia de vida requieren de un análisis de contenido (Ghiglione, Beauvois, Chabrol y Trognon, 1980; Pujadas, 1992; Del Río Sadornil, 1998) y ello consiste en reducir la información en categorías según las variables que orientan el trabajo (...). Desde aquí, la categorización consiste en agrupar las unidades que son similares en un mismo tópico o temática (Cortés y Medrano, Op. cit.: 66-67).

En este trabajo de investigación se hizo tal como sugiere la cita. Los datos se fueron reduciendo mediante el agrupamiento de evidencias que tenían vínculos en torno a una materia (Rodríguez, Gil y García, 1996). El árbol de categorías se fue conformando teniendo en cuenta las similitudes de contenido y las relaciones existentes entre las informaciones. Al mismo tiempo se intentaba ser sensible a los distintos matices expresados en cada fragmento. Todo ello resultaba clave a la hora de armar una estructura que debía permitir el análisis holístico y minucioso de la realidad sujeta a estudio. La estructura conformada por el entramado de categorías debía proporcionar la oportunidad de acceder a cualquier aspecto susceptible de análisis en toda su complejidad.

El esquema analítico se fue conformando de manera progresiva. El proceso de síntesis de la información y constitución del listado de categorías requirió que se diesen los siguientes pasos (en el orden que se presentan):

- Unidades de Análisis

- Tematización

- Bloques Temáticos

- Categorías.

\subsubsection{Unidades de Análisis}

Como ya se dijo en el apartado 'Categorización de la Información. Inicio', la Unidad de Análisis (UA) son el segmento de información sustantiva más básico susceptible de ser escudriñado en la investigación. Y constituye el primer avance en la construcción del árbol de categorías.

En esta investigación se elaboraron dos listados de UA, uno por cada bloque del relato biográfico: uno a partir del Relato en primera persona (R1) y el otro a partir del Relato en tercera Persona (R3). La compilación de ambos listados era recogida en un documento que en total sobrepasaba las 50 páginas. Haciendo la suma de todas las Unidades de Análisis localizadas, en total se destilaron de la biografía un total de 712 UA y 64 fotografías. Concretamente, del 'Relato en primera persona' (R1): 273 UA y 64 fotografías, y del 'Relato en tercera persona' (R3): 439 UA.

\subsubsection{Tematización}

La tematización, o establecimiento de los temas, comprendió el segundo paso en el proceso de reducción de la información. Todas las unidades de análisis fueron revisadas minuciosamente con el propósito de materializar una relación de todos los asuntos comprendidos en ellas. Dichos asuntos fueron ordenados por materias.

Cada tema podía dar cabida a cuantas UA estuviesen relacionadas con el asunto que representaba. Véase un ejemplo: 


\section{Tema: CI.1. Ahmed traía la educación de casa}

UA: $\quad$ 1.- "Él se fue con la educación de la casa". El padre era la persona de referencia de la familia y era rígido con las normas que él establecía. (Cap. I. Relato en $3^{\mathrm{a}}$ persona).

13.- Informe Inicial Arteria. La tutora considera a sus padres responsables del nivel educativo de Ahmed. (Cap. VII. Relato en $3^{\text {a }}$ persona).

33.- No olvides que el primer factor básico a la hora de valorar la inclinación de un chaval en su vida delincuencial es la familia, y si ahí te encuentras un panorama muy negativo, si no hay una estructura normativa de base, si no hay una estructura de arrope de base difícil lo tiene, porque volvemos a lo mismo, no puedes dar lo que no tienes y si no has recibido, en muchas ocasiones, el afecto, la norma, el control, es decir, un esquema familiar claro, es muy difícil. (Cap. VIII. Relato en $3^{\mathrm{a}}$ persona).

La tarea, por tanto, era compleja, porque no sólo se trataba de hacer un listado con los asuntos que se destilaban de cada UA, sino que además de ello, había que ir ordenándolas en función de los elementos en común que existían entre ellas.

Teniendo en cuenta la enorme cantidad de información registrada, la tematización se llevó a cabo siguiendo el mismo procedimiento que durante la primera fase del proceso de categorización, es decir, de manera independiente en cada relato (R1a y R3a).

De la tematización del 'Relato en la persona' se destilaron 80 temas, con sus correspondientes subtemas. Se trató de mejorar la capacidad de síntesis durante la tematización del 'Relato en 3a Persona', especialmente, en lo concerniente a la organización de la información. De aquella segunda parte de la biografía emergieron 40 temas, por supuesto, con sus correspondientes subtemas, sub-subtemas y sucesivos. En aquel segundo ejercicio de tematización parece que se empezaron a relacionar un poco mejor los distintos asuntos.

Para reconocer con claridad los temas pertenecientes a uno u otro relato se enumeraron (con números romanos) correspondientemente, haciendo una distinción entre ellos: en la parte correspondiente a la versión en primera persona la numeración contempló del I al LXXX y los pertenecientes a la narrativa en tercera persona comenzaron a partir del C.

\subsubsection{Bloques Temáticos}

La siguiente etapa de aquel proceso de síntesis era el establecimiento de bloques temáticos. Como se puede imaginar, consistía en la agrupación de temas con alguna característica en común. Al igual que en la fase anterior, en aquel estadio había que ordenar con sentido dichos bloques, sub-bloques y sucesivos, definiendo y consolidando la compleja estructura que había de dar cobertura analítica a toda aquella información.

Armar la estructura era probablemente la tarea más compleja del proceso de categorización. El trabajo de síntesis y organización de la información era enormemente laborioso, sobre todo, porque la cantidad de datos era muy abundante. En consecuencia, los bloques temáticos se seguían desarrollando separadamente para el 'Relato en 1a persona' y el 'Relato en 3a persona'.

A partir de este estadio se empezaba a vislumbrar un cuerpo de análisis común (había muchas similitudes entre los bloques temáticos destilados del 'relato en $1^{\text {a }}$ persona' y del 'relato en $3^{\text {a }}$ persona'). Definir sendos bloques temáticos fue una tarea enormemente laboriosa que, además de una incalculable cantidad de horas de trabajo, conllevó muchos ensayos, 
pruebas, cambios y modificaciones. La definición de aquella estructura fue un logro fundamental de cara al establecimiento definitivo de las categorías de análisis.

\subsubsection{Categorías de Análisis}

En el cuarto paso del proceso de categorización se definió el listado de categorías de análisis. No se considera necesario compartir dicho listado, pero sí relacionar, a continuación, sus aspectos más destacados:

- En primer lugar, se establecieron cinco categorías transversales (I. Características Personales de Ahmed, II. Religión, III. Delincuencia, IV. Inmigración, V. Identidad), que atravesaban el caso y que, en consecuencia, había que tener en cuenta a lo largo de todo el proceso de análisis.

- Atendía a los tres momentos que originalmente se habían distinguido en el biograma (antes del arresto, el proceso de reforma juvenil y tras la finalización de las medidas). El planteamiento se realizó poniendo el foco en tratar de comprender qué trascendencia había tenido el mencionado proceso de reforma en su vida.

- Se identificaron las vivencias sociales y pedagógicas que Ahmed experimentó durante las dos medidas judiciales que dieron cuerpo al mencionado proceso de reforma: la primera, 'Internamiento en centro', y la segunda, 'Libertad vigilada'. Bajo sendas medidas tuvieron lugar una serie de experiencias, de distinta índole, que influyeron en el desarrollo de su historia. En el siguiente esquema se pueden ver todas ellas de un modo gráfico:

Figura 1. Experiencias pedagógicas y sociales que se pusieron en juego durante el proceso de reforma juvenil de Ahmed

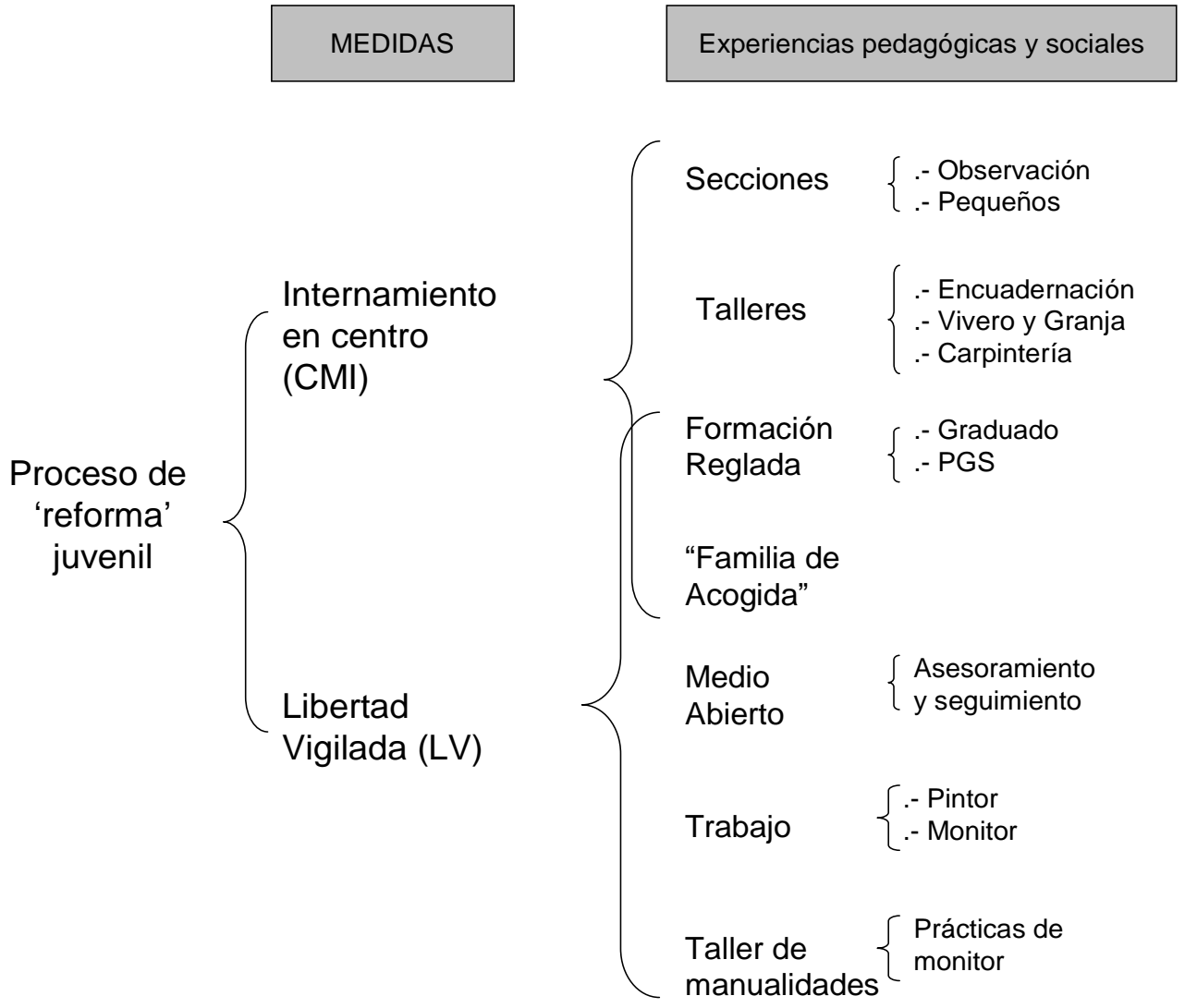


Elaboración propia

Las categorías que finalmente se definieron en el listado de 'Categorías de análisis' se consideraron categorías "iniciales" (no finales) porque habían de ser interpretadas como puntos desde los que poder zambullirse y bucear en la información recolectada. En ningún caso eran fijas, inamovibles o rígidas, por el contrario, se consideraron una plataforma de claves para empezar a interpretar aquella realidad. Aquellas categorías ayudaron a iniciar el análisis y durante el proceso se fueron definiendo y precisando a partir de lo que iban señalando los datos.

\subsection{Definición del foco de la investigación}

La investigación se inició a partir de un puñado de preguntas y dudas que el investigador se planteaba en torno al caso, pero sin tener de partida un foco de investigación definido. Dicho foco de investigación se fue forjando a lo largo de todo el trabajo de investigación y llegó a definirse de manera precisa al término del proceso de categorización.

En concreto, el foco se estableció sobre las claves que contribuyeron a que Ahmed pasase de "menor infractor" a convertirse en "educador de menores". ¿Qué elementos se conjugaron y/o confluyeron para que aquel joven experimentase aquella transformación? En concreto, se quería saber qué se hizo desde un punto de vida pedagógico y social (tanto en la medida de internamiento en centro como en la de libertad vigilada), para que aquel caso evolucionara de aquella manera.

Además de escudriñar, una por una, las distintas experiencias presentadas anteriormente en el esquema de llaves, se pretendía vislumbrar las relaciones establecidas entre ellas, para ver cómo redundaban en el desarrollo de cada medida y del proceso de reforma en general.

Desarrollar aquel análisis, también quería arrojar luz dentro de los procesos de reforma, pues apenas se encontraron investigaciones que estudiasen la materialización de las medidas desde su interior ni desde esa perspectiva. En ese sentido, se pensó que realizar aquel análisis permitiría entrar en las instituciones encargadas de operativizarlas y visibilizar cómo se llevan a cabo. Hay un enorme desconocimiento en torno al trabajo que, sociopedagógicamente, se desarrolla con el/la "menor infractor/a", por lo que el trabajo pretendía contribuir a mejorar el conocimiento de esa realidad. Parece que va siendo hora de que se empiece a naturalizar la situación de los/as "menores infractores" y que comencemos a humanizar lo que con esos/as muchachos/as se hace, porque, en definitiva, lo que se pretende es tratar de recuperarlos socialmente y darles una segunda oportunidad como personas. Por último, dar a conocer dichas prácticas pretende iluminar experiencias que pueden servir de referente a otros/as profesionales.

Todas estas ideas, que en última instancia conformaron el foco de la investigación, se fueron definiendo en el recorrido realizado por los distintos estadios que conformaron el proceso de investigación. Como consecuencia, se definió un foco de investigación bastante preciso porque se fue configurando de un modo emergente a partir del escrutinio al que se sometió el sustrato empírico a lo largo de todo aquel trayecto.

\section{Conclusiones}

Bertaux dice que "la investigación es una tarea colectiva y acumulativa en la que cada trabajo realiza su propia contribución" (2005: 18). A continuación, compartimos algunas de las contribuciones metodológicas más significativas que la historia de vida realizada en torno al caso de Ahmed hace a la investigación biográfica: 
- En este trabajo se han desarrollado dos instrumentos metodológicos que se han revelado fundamentales dentro de la investigación biográfica: Las Unidades Biográficas (UB) y las Unidades de Análisis (UA). A continuación, se definen de manera concisa:

- Unidad Biográfica (UB): Selección de información de interés narrativo. Comprende datos que a priori podrían ayudar a construir el relato. La narrativa debe ser elaborada conjugando las diferentes UB establecidas. La biografía resultará como producto de su tratamiento y organización.

- Unidad de Análisis (UA): Esta unidad identifica información sustantiva que es susceptible de ser analizada de acuerdo con el objeto de la investigación. Cada UA comprende algún dato de interés para el estudio. Es el elemento que se tiene en cuenta en primer lugar a la hora de elaborar el árbol de 'categorías de análisis'. En ellas se encuentran las evidencias que serán usadas durante la interpretación de los datos.

Cada Unidad de Análisis tiene su homóloga Unidad Biográfica, sin embargo, no ocurre lo mismo al contrario, no toda Unidad Biográfica se traduce, necesariamente, en una Unidad de Análisis.

- Desde un punto de vista narrativo, el modelo de 'Relatos Cruzados' es mucho más ágil y dinámico que cuando se cruzan los distintos testimonios de manera 'no consecutiva'. Sin embargo, esta última alternativa ofrece una posibilidad a aquellos casos en los que la implementación tradicional del modelo no resulta posible. Se aplica en situaciones como la experimentada en este trabajo, en un estudio inductivo donde la cantidad de información es ingente y no se tiene certeza de qué información puede ser descartada de cara a la elaboración de la biografía.

Desde una perspectiva antropológica y/o sociológica la variante en esta investigación creada tiene un valor importante, porque se trata de historias que poseen cuantiosa información.

- La biografía no tiene, necesariamente, que ser elaborada antes de realizar el análisis de los datos (como se hizo en esta investigación). Puede ser redactada tras el examen de la información. De hecho, puede que hacerla en este segundo momento permita el desarrollo de una narración más ajustada al foco de estudio.

- Las categorías de análisis deben ser entendidas teniendo en cuenta el momento de la investigación en el que se desarrollan y, en consecuencia, vistas de una manera más abierta y flexible de lo que habitualmente se hace. Dichas categorías no deben encorsetar al investigador dentro de una(s) idea(s) e impedirle ver más allá de ella(s). Es justo todo lo contrario. Las categorías son trampolines que deben permitir al investigador zambullirse en cualquier punto del océano de datos al que se enfrenta. Y proporcionarle suficiente impulso como para profundizar hasta donde sea necesario. Todo ello, para comprender la complejidad del caso y descifrar sus verdaderos enigmas. Pero, el investigador no sólo habrá de sumergirse en toda esa información, sino que, fundamentalmente, tendrá que examinarla. Por eso, a las categorías se les ha de otorgar un carácter inicial, porque permiten el inicio del análisis, pero todo el proceso de escrutinio que ese estadio requiere es mucho más complejo.

Como consecuencia de lo expuesto, se considera que las categorías definitivas son los títulos de los diferentes apartados, subapartados y sucesivos del informe que comprende los resultados (la interpretación y cuestionamiento de los datos), porque son los que en última instancia dibujan, de manera holística, la estructura del análisis que se ha realizado sobre el caso, teniendo en cuenta todas sus aristas. 
- En una investigación inductiva el foco de estudio se determina progresivamente durante los distintos estadios que se recorren, lo que permite que el foco de investigación vaya emergiendo y se vaya definiendo de manera precisa y con sentido en virtud de la información que contenga el sustrato empírico. La manera en la que los datos fueron procesados y organizados en esta investigación (tuvieron que pasar diferentes estadios y fueron tratados de manera meticulosa), animando su revisión constantemente, permitió dicha definición.

\section{Referencias bibliográficas}

Arnaus, R. (1995). Voces que cuentan y voces que interpretan: Reflexiones en torno a la autoría narrativa en una investigación etnográfica. En J. Larrosa, R. Arnaus, V. Ferrer, N. Pérez de Lara, F.M. Connelly, D.J. Clandinin et al. Déjame que te cuente. Ensayos sobre narrativa y educación (61-78). Barcelona: Ediciones Alertes.

Bertaux, D. (2005). Los relatos de vida. Perspectiva etnosociológica. Barcelona: Edicions Bellaterra.

Bofill Tortosa, E.J.; Penacho Gómez, A.M. y Quinzá Lleó, X. (2006). Vidas ejemplares. Relatos de chavales enredados entre el sistema de protección y reforma. Aragón: Ikusbide.

Bolívar, A.; Domingo, J. y Fernández, M. (2001). La investigación biográfico-narrativa en educación. Enfoque y metodología. Madrid: La Muralla.

Cortés, A. y Medrano, C. (2007). Las historias de vida: fundamentación y metodología. En C. Medrano (coord.). Las historias de vida. Implicaciones educativas (47-80). Buenos Aires (Argentina): Alfagrama ediciones.

Gamella, J.F. (1990). La historia de Julián. Memorias de heroína y delincuencia. Madrid: Popular.

González, M.F. y Padilla-Carmona, M.T. (2014). Investigación narrativa: las historias de vida. En B. Ballesteros Velázquez (coord.). Taller de investigación cualitativa (77102). Madrid: UNED.

González Monteagudo, J. (2010). Biografía, Identidad y Aprendizaje en estudiantes universitarios no tradicionales. Estudio de caso de una mujer trabajadora. Profesorado: Revista de currículum y formación del profesorado, Vol. 14, n 3, 131147.

Kushner, S. (2002). Personalizar la evaluación. Madrid: Morata.

Lewis, O. (2012). Los hijos de Sánchez. Autobiografía de una familia mexicana. Una muerte en la familia Sánchez. México: FCE-Fondo de Cultura Económica.

Pujadas Muñoz, J.J. (2002). El método biográfico: El uso de las historias de vida en ciencias sociales. Madrid: Centro de Investigaciones sociológicas. 
Rivas Flores, J.I., Herrera Pastor, D., Jiménez Cortés, R. y Leite Méndez, A. (2009). Conocer la escuela a través de nuestras vidas. La biografía escolar como estrategia de aprendizaje. En A. García-Valcárcel (Ed.). Experiencias de innovación docente Universitaria (507-512). Salamanca: Ediciones Universidad de Salamanca.

Roberts, B. (2002). Biographical research. Berkshire (Inglaterra): Open University Press.

Rodríguez Gómez, G.; Gil Flores, J. y García Jiménez, E. (1996). Metodología de la investigación cualitativa. Málaga: Aljibe.

Waller, R. y Simmons, J. (2009). Vidas a través de la lente de un ojo de buitre: Interpretando cuentos de aprendices. En J.I. Rivas Flores y D. Herrera Pastor (coords.). Voz y educación. La narrativa como enfoque de interpretación de la realidad (55-74). Barcelona: Octaedro. 